(C) 2001 IEEE. Reprinted, with permission, from King, D.J., Bradley, D.A., Mansoor, S.P., Jones, D.I., Aris, F.C. and Jones, G.R. Using a fuzzy inference system to control a pumped storage hydro plant. In: Proceedings of the loth IEEE International Conference on Fuzzy Systems, Melbourne, Australia, 2-5 December 2001, Vol.3. Piscataway, NJ: IEEE. pp.1008-1011. 2001.

This material is posted here with permission of the IEEE. Such permission of the IEEE does not in any way imply IEEE endorsement of any of the University of Abertay Dundee's products or services. Internal or personal use of this material is permitted. However, permission to

reprint/republish this material for advertising or promotional purposes or for creating new collective works for resale or redistribution must be obtained from the IEEE by writing to pubs-permissionsdieee.org.

By choosing to view this document, you agree to all provisions of the copyright laws protecting it. 


\title{
USING A FUZZY INFERENCE SYSTEM TO CONTROL A PUMPED STORAGE HYDRO PLANT
}

\author{
D.J. King \& D.A. Bradley, \\ The University of Abertay Dundee \\ Dundee, Scotland, UK
}

\author{
S. Mansoor \& D. Jones \\ The University of Wales; Bangor \\ Bangor, Wales, UK
}

\author{
F.C. Aris \& G. Jones \\ First Hydro Ltd. \\ Dinorwig Power Station,Wales, UK
}

Contact author: D.J. King, School of Science and Engineering, University of Abertay, Bell Street, Dundee, Scotland, UK, DD1 1HG.

Phone: +44 (0) $1382308198 \quad$ Fax: +44 (0) 1382308261

e-mail: d.j.king@abertay.ac.uk

Keywords: Fuzzy Inference Systems, Control Applications.

Technical Area: T4

\begin{abstract}
The paper discusses the development of a Fuzzy Inference System (FIS) based governor control for a pumped storage hydroelectric plant. The First Hydro Company's plant at Dinorwig in North Wales is the largest of its kind in Europe and is mainly used for frequency control of the U.K. electrical grid. In previous investigations, a detailed model of the plant was developed using MATLAB ${ }^{\circledR} /$ SIMULINK $^{\circledR}$ and this is now being used to compare FIS governor operation with the Proportional-Integral-Derivative (PID) controller currently used. The paper describes the development of an FIS governor, and shows that its response to a step increase in load is superior to the PID under certain conditions of load. The paper proceeds to discuss the implications of these results in view of the possible practical application of an FIS governor at the Dinorwig plant.
\end{abstract}

\section{INTRODUCTION}

First Hydro Ltd operate the largest pumped storage hydroelectric plant in Europe comprising six 313MW reversible pump-turbines. . Due to its ability to respond quickly to changes in power demand, its primary role is frequency control and it is ideally suited to supplying the power make-up required to maintain system. frequency under varying load conditions. The current governor is essentially a PID controller implemented digitally on Programmable Logic Controllers and acts to control the response of the turbines to changes in frequency. The system frequency error signal is fed back to the PID controller, and the individually adjustable proportional, integral and derivative gains determine the initial rise time, overshoot and settling time of the power output. There are however significant operational advantages to be gained through an improvement on the response produced by the current governor, particularly in respect of the ability to respond to major load changes.

The research uses a MATLAB ${ }^{\circledR} /$ SIMULINK $^{\circledR}$ model of the Dinorwig plant developed at the University of Wales, Bangor (1) to enable a comparison between the behaviour of the PID governor with the FIS implementation. It has been shown in other areas that Artificial Intelligence (AI) techniques, such as Fuzzy Logic (FL), Artificial Neural Networks (ANN) and hybrids of the two techniques are able to adapt and learn to model complex, non-linear systems (2-6). Indeed, control of a single hydroelectric generator using a hybrid FL/ANN approach has been reported $(7,8)$, but these applications are not concerned with frequency control. There is also available a significant body of work aimed at first replicating, and then improving on, PID type control using FL controllers $(9,10)$. It was therefore decided to evaluate the use of FL techniques as a replacement for the PID governor providing enhanced performance.

\section{CASE STUDIES}

The station model was initially set up so as to look at a system with just one turbogenerator unit operating in frequency control mode and to assess its response to a step increase in load on a 30GW grid. The simulation was set up so that the system was initially balanced when a step increase in load of $0.5 \mathrm{pu}(150 \mathrm{MW})$ was then introduced, this being the largest increase of load that a single unit operating in frequency control with a base 
power setting of 150MW (half-load) is able to pick up. A number of different FIS's were initially built using the MATLAB ${ }^{\circledR}$ Fuzzy Logic Toolbox (11), enabling the following four cases to be investigated:

Case 1 - Turbogenerator unit with PID control.

Case 2 - Turbogenerator unit with Fuzzy 1 control.

Case 3 - Turbogenerator unit with Fuzzy 2 control.

Case 4 - Turbogenerator unit with Fuzzy 3 control.

In Case 1, the PID had the following settings:

$\begin{array}{llr}\text { Proportional gain } & - & 10 \\ \text { Integral gain } & - & 12 \\ \text { Derivative gain } & - & 2\end{array}$

These are the standard settings used at Dinorwig for frequency control. In addition, a droop setting of either $4 \%$ or $1 \%$ would be included as part of the controller to limit the increment of power that could be delivered by a single machine. However, during the initial investigation into the use of an FIS to control the generator, the effect of the droop was not taken into account. In Case 2, the first FIS model, Fuzzy 1, uses a single input variable, the frequency error $(\Delta f)$. For Case 3, the Fuzzy 2 model uses two input signals, $\Delta f$ and the rate of change of frequency $(\mathrm{d} f / \mathrm{d} t)$. For Case 4 , the Fuzzy 3 model uses $\Delta f$ and the output power error signal $\Delta \mathrm{P}$ as the input signals.

\subsection{Case 1}

The simulation was run under the conditions described above and a plot of frequency $(f)$, generator output power $\left(\mathrm{P}_{\text {OUT }}\right)$ and load power $\left(\mathrm{P}_{\mathrm{L}}\right)$ derived as shown in Fig. 1. Initially, $\mathrm{P}_{\mathrm{L}}$ matches $\mathrm{P}_{\mathrm{OUT}}$ at $150 \mathrm{MW}$, and hence frequency $(f)$ is stable at $50 \mathrm{~Hz}$. At 20 seconds into the simulation there is a step increase in $\mathrm{P}_{\mathrm{L}}$ of $150 \mathrm{MW}$ to which the generator responds by increasing output which, without the inhibitory effect of droop, approaches the demand load power, reaching 295MW after a further 60 seconds.

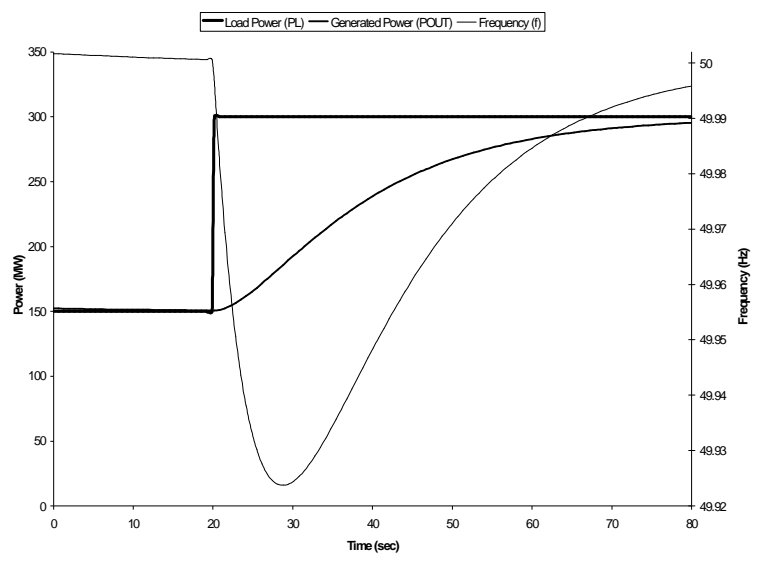

Figure 1: Plots of power and frequency for PID controller with 0.5 p.u. load increase

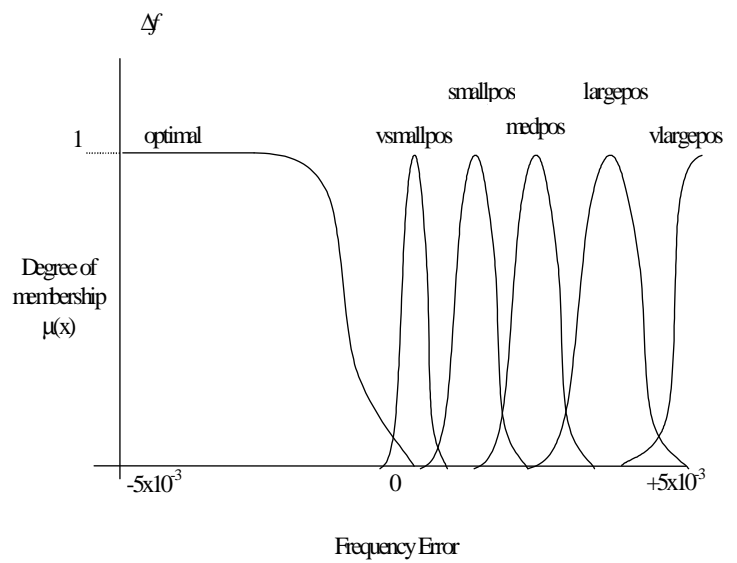

Figure 2: Universe of discourse for $\Delta f$ membership functions (Case 2)

\subsection{Case 2}

After several attempts the Fuzzy 1 model was developed using a set of six fuzzy rules, as shown below:
Rule 1
IF $\Delta f$ is optimal THEN $\alpha$ is optimum.
Rule 2 IF $\Delta f$ is vsmallpos THEN $\alpha$ is vsmallpos.
Rule 3 IF $\Delta f$ is smallpos THEN $\alpha$ is smallpos.
Rule $4 \quad$ IF $\Delta f$ is medpos THEN $\alpha$ is medpos.
Rule $5 \quad$ IF $\Delta f$ is largepos THEN $\alpha$ is largepos.
Rule 6 IF $\Delta f$ is vlargepos THEN $\alpha$ is vlargepos.

Where $\alpha$ is the output signal to the guide vane, which controls the flow of water through the turbine, and hence the mechanical power into, and the electrical power from, the generator. 


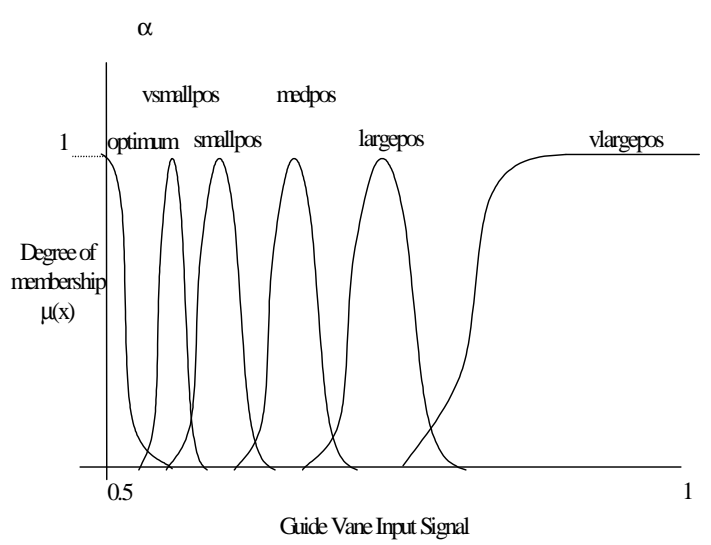

Figure 3: Universe of Discourse for $\alpha$ membership functions (Case 2)

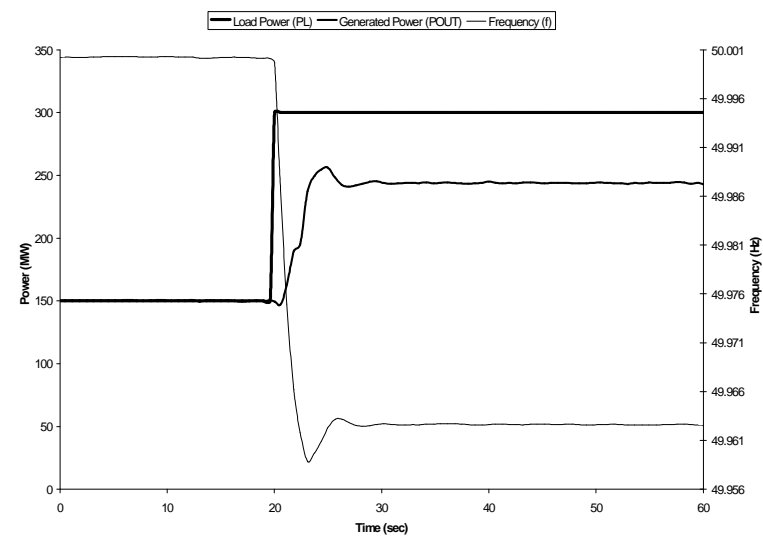

Figure 4: Plots of power and frequency for Case 2 with 0.5 p.u. load increase

The Universe of Discourse and the Membership Functions (MF) for $\Delta f$ and $\alpha$ are shown in Figs. 2 and 3. Note that $\alpha$ is limited to values between 0.5 and 1 . The lower limit coincides with the minimum output power of 150MW set for units in frequency control mode. The Smallest of Maximum defuzzification function was then used which ensured that the minimum value for $\alpha$ would be 0.5 .

The initial results from this model were not as good as those with the PID governor. Efforts were then made to improve the response by moving the MF's and changing their widths, but with little effect. It was then realised that the value for $\Delta f$ was not reaching its nominal maximum value and as a result, the rules governing the higher values of $\alpha$ were not being fired. The next move was to therefore to reduce the maximum value of $\Delta f$. When this was done, the results were as shown in figure 4 . The power output falls short of the $300 \mathrm{MW}$ load value. Hence the final frequency value is only $49.963 \mathrm{~Hz}$. However, the initial response of the FIS is much better than the PID, reaching its final value within 10 seconds. of the disturbance. It was noticed that although the limits for $\Delta f$ had been reduced, the rule governing the maximum value for $\alpha$ was still not being fired. This was because the value of $\Delta f$ decreases as the frequency gets closer to $50 \mathrm{~Hz}$, and therefore it can never reach the maximum value and fire the rule governing maximum $\alpha$. It appeared that it was not possible to make much further improvement by using $\Delta f$ as the only input to the FIS.

\begin{tabular}{c|c|c|c|c|c|c|}
\cline { 3 - 7 } \multicolumn{1}{c|}{} & okay & vsmallpos & smallpos & largepos & vlargepos \\
\cline { 2 - 7 } & negative & vsmallpos & vsmallpos & vsmallpos & smallpos & largepos \\
\cline { 2 - 7 } $\mathrm{d} f / \mathrm{d} t$ & okay & okay & smallpos & smallpos & largepos & vlargepos \\
\cline { 2 - 7 } & positive & okay & okay & largepos & vlargepos & vlargepos \\
\cline { 2 - 6 } & &
\end{tabular}

Figure 5: Fuzzy Associative Memory map for Case 3.

\subsection{Case 3}

As stated, this case involves the use of the two input variables $\Delta f$ and $\mathrm{d} f / \mathrm{d} t$. After experimentation with different rules, MF's and defuzzification functions the Fuzzy 2 model was developed. The Fuzzy Associative Memory (FAM) map covering the 15 rules for this model are shown in Fig. 5. The FIS based on this FAM was built using similar shaped MF's to those used in Case 2. Also, for the fuzzy logic operations the product AND fuzzy logic operators were used, as opposed to the more usual minimum AND as used in Case 1. The Centroid defuzzification function was also used, this is the most widely used in FIS applications as it tends to give a smoother output. Several attempts were made to improve the output from this FIS with significant improvements achieved by changing the limits for the $\Delta f$ input while the slopes of the MF's were steepened to stop oscillations caused by different rules firing. After much tinkering the best result is shown in figure 6 . As can be seen, the final value of $f$ has improved to $49.974 \mathrm{~Hz}$. This FIS was tested for a step increase of 0.3 p.u. to compare the response, and the results are shown in Fig. 7.

Using this model, a final value for $f$ of $49.997 \mathrm{~Hz}$. is achieved although the output has slight oscillations on it. As a comparison a simulation was run for the same step increase in load with the PID controller, the results of which are shown in Fig. 8. This shows that the FIS has improved on the PID governor's rise time, although the latter's output is much smoother. 


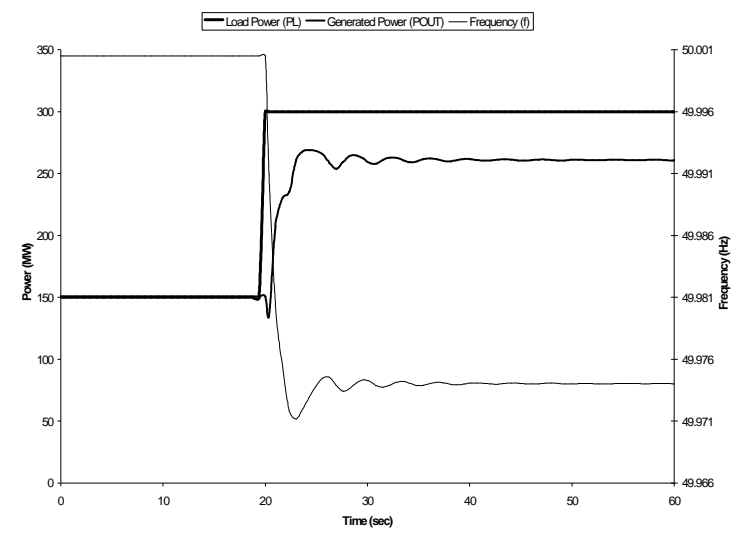

Figure 6: Plots of power and frequency for Case 3 with 0.5 p.u. load increase

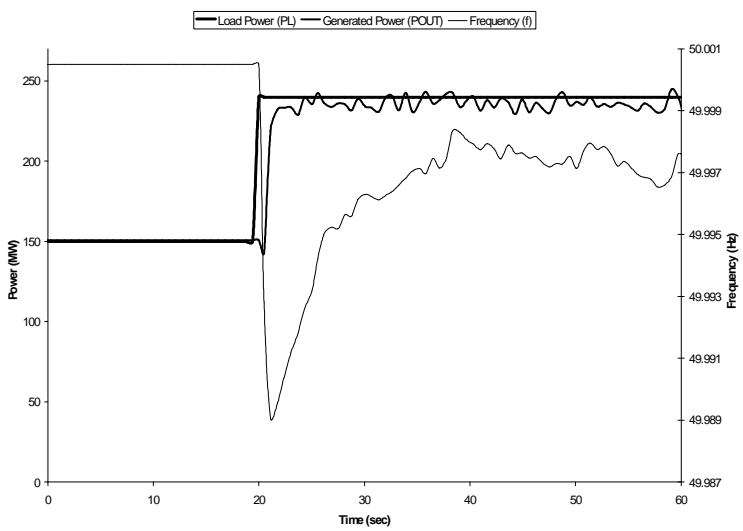

Figure 7: Plots of power and frequency for Case 3 with 0.3 p.u. load increase

Using this model, a final value for $f$ of $49.997 \mathrm{~Hz}$. is achieved although the output has slight oscillations on it. As a comparison a simulation was run for the same step increase in load with the PID controller, the results of which are shown in Fig. 8. This shows that the FIS has improved on the PID governor's rise time, although the latter's output is much smoother.

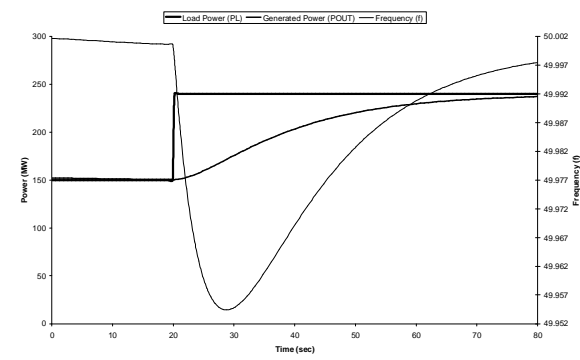

Figure 8: Plots of power and frequency for PID controller with 0.3 p.u. load

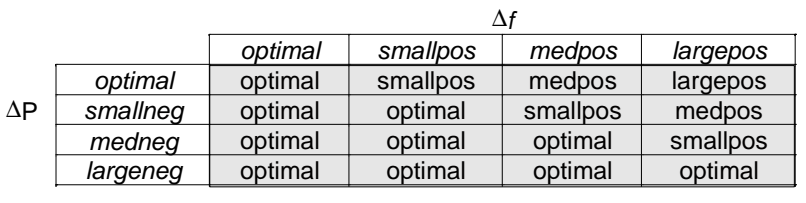

Figure 9: Fuzzy Associative Memory map for Case 4

\subsection{Case 4}

While trying to improve the output in the previous cases it was realised that it was difficult to get the FIS to fire the rule governing the highest value for $\alpha$. As previously mentioned, this is because the input signal to $\Delta f$ gets smaller as the output gets closer to $50 \mathrm{~Hz}$. However, for a step increase in load of $0.5 \mathrm{p} . \mathrm{u}$., $\Delta \mathrm{P}$ must stay at $150 \mathrm{MW}$ if the output power is to match the load. Therefore, $\Delta \mathrm{P}$ could be used in conjunction with $\Delta f$ as inputs to the FIS to ensure that the rule governing the maximum value for $\alpha$ is fired. After several tries the Fuzzy 3 model with 11 rules was developed as shown in the FAM of Fig. 9.

After trying a number of variations of limit values for $\Delta f$ and $\Delta \mathrm{P}$, and changing the position and form of the MF's (which were Gaussian in shape) the output shown in Fig.10 was obtained. This shows that with this particular configuration, the frequency is forced back to $50 \mathrm{~Hz}$. The same FIS was also tested for a step increase of power of 0.3 p.u. This time the frequency recovers to $49.96 \mathrm{~Hz}$. It was noticed that the best way to get the highest output from the FIS was to make sure that $\Delta \mathrm{P}$ was at its maximum when $\Delta f$ was reduced. An effort was therefore made to

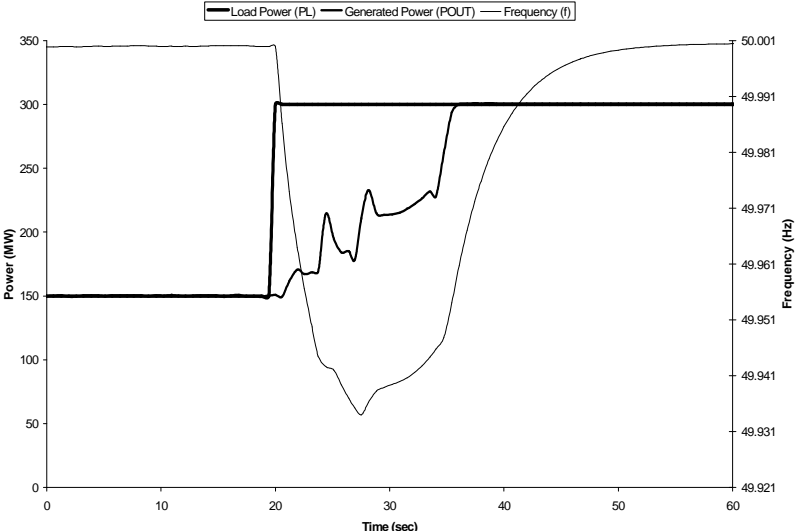

Figure 10: Plots of power and frequency for Case 4 with 0.5 p.u. load increase. simplify the number of rules by saying that: 
Rule 1 If $\Delta f$ is optimal $\mathrm{OR} \Delta \mathrm{P}$ is optimal THEN $\alpha$ is optimal

Rule 2 If $\Delta f$ is largepos $\mathrm{OR} \Delta \mathrm{P}$ is largeneg THEN $\alpha$ is largepos

Where optimal and largepos for $\alpha$ would have to be as close to 0.5 and 1.0 as possible. Rule 1 ensures that the output power is kept to $150 \mathrm{MW}$ when required, while Rule 2 makes sure that $\alpha$ is kept to a maximum for a large value of $\Delta \mathrm{P}$, even when $\Delta f$ is reduced. More rules would be needed to give suitable $\alpha$ values for power changes between those limits. In the event there was only one additional rule added:

Rule 3 If $\Delta \mathrm{f}$ is medpos AND $\Delta \mathrm{P}$ is medneg THEN $\alpha$ is medpos

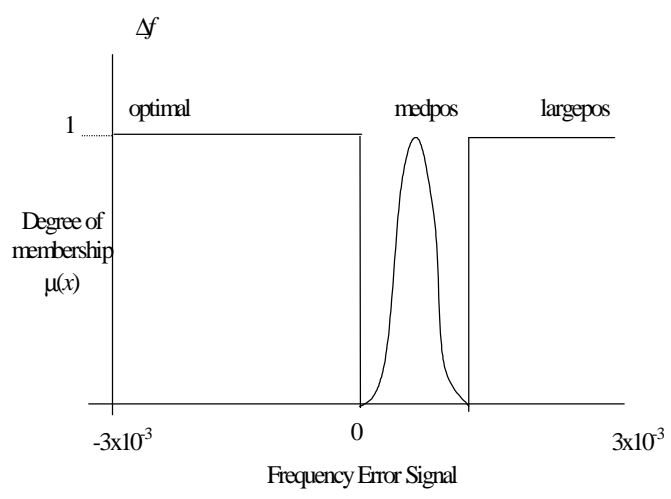

Figure 11: Universe of Discourse for $\Delta f$ membership functions (Amended Case

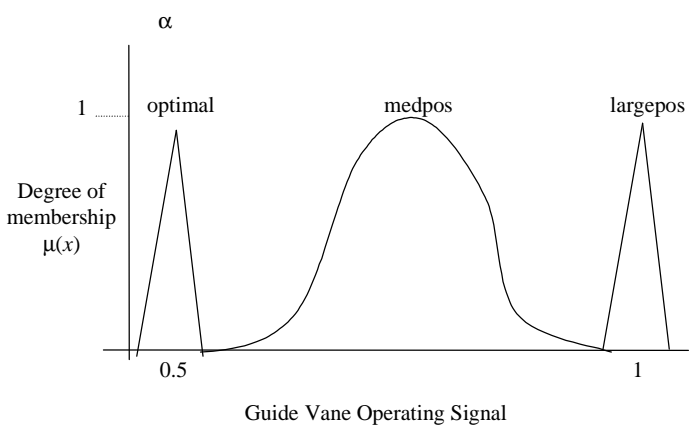

Figure 13: Universe of Discourse for $\alpha$ membership functions (Amended Case 4)

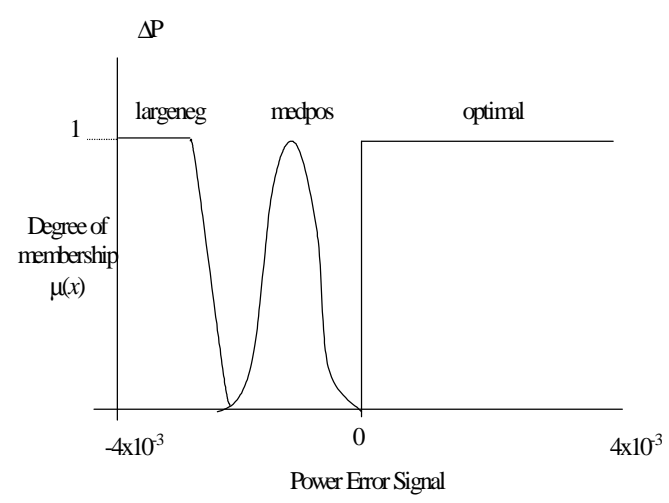

Figure 12: Universe of Discourse for $\Delta P$ membership functions (Amended Case 4)

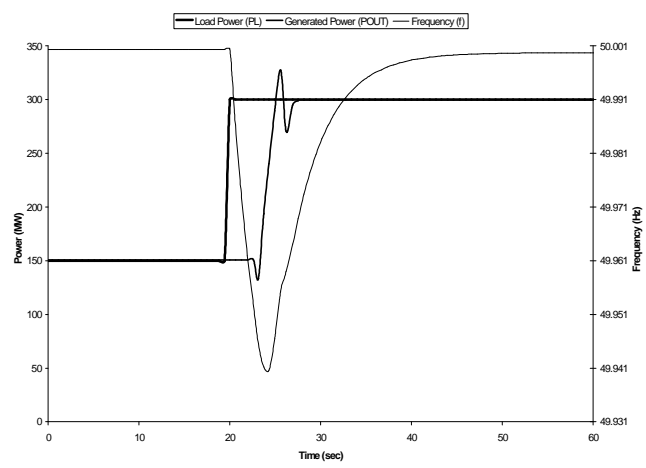

Figure 14: Plots of power and frequency for amended Case 4 with 0.5 p.u. load increase

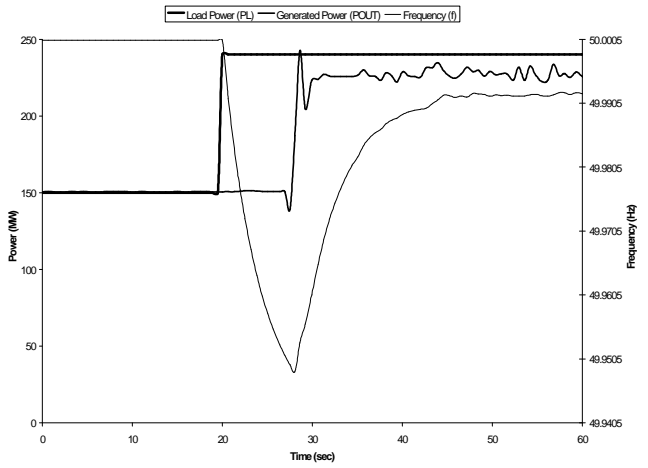

Figure 15: Plots of power and frequency for amended Case 4 with 0.3 p.u. load increase

MF's for $\Delta f, \Delta \mathrm{P}$ and $\alpha$ were developed as shown in Figs. 11, 12 and 13. After some minor modifications of the basic MF's, to avoid oscillations between rules, the results for a step change in load of 0.5 p.u. and 0.3 p.u. are shown in figures 14 and 15 respectively. Figure 14 shows that for 0.5 p.u. step increase in load the frequency 
recovers to $50 \mathrm{~Hz}$ and the output is very smooth. In figure 15 , although the output is noisy for the 0.3 p.u. load increase, the frequency also recovers to a final value of just over $49.99 \mathrm{~Hz}$.

\section{CONCLUSIONS AND FURTHER WORK}

It can be seen from all the above cases that in comparison with the PID controller, the FIS governor made a significant improvement to the initial response by considerably reducing the rise time. However, to get the best results took a lot of manipulation and 'tuning' of the shape, position and type of MF's used, and also depended on the number of fuzzy rules and which logical operators were used as well as on which defuzzification function was used. There were therefore a lot of factors that could be changed for any given case and thus a lot of time was taken up trying to find the optimum solution.

As already mentioned, there was a particular problem involved in building an FIS governor based solely on changes in frequency. This was due to the fact that as the frequency returned to the nominal value, the frequency error signal was reduced, making it difficult to ensure that the rule governing the maximum output to the guide vane was fired. Although the MF's could be adjusted to get the required result, this would make the FIS very inflexible, and unable to respond correctly for different loads. Including the rate of change of frequency did help, but it was very difficult to get the balance right between the sensitivity of the output to the two inputs and a smooth response without oscillations.

Using a combination of the frequency error signal and the power error signal gave the best results as the FIS could be set up so that $\Delta \mathrm{P}$ could fix the output to the correct value once $\Delta f$ has returned to a small value. Even so, as seen in Fig. 15, when the load step was reduced, the FIS had difficulty in giving a smooth output and thus more tuning was needed. This leads to the possibility that it may not be feasible to get a good response for all possible load values and that further rules may be required to get a better response over a wide range of load conditions.

It has been shown that for a given step increase in load the FIS can be set up to give an excellent response. A possible strategy may therefore be to have several FIS's set up to respond to various load increases. Some form of pre-processor, possibly an ANN, could then be used to select the appropriate FIS for given operating conditions. However, setting up the individual FIS's to respond as required to the step increase needed a great deal of adjustment to factors such as the size, shape and position of the MF's, the type of defuzzification function used, the number of fuzzy rules and the value of the limits on each individual Universe of Discourse. Therefore a strategy involving some form of sensitivity analysis, to identify which changes have most effect on the output, could reduce the time taken to develop the FIS. In this respect some form of Taguchi sensitivity analysis using orthogonal arrays may be advantageous (12).

Alternatively, it may be possible to develop an FIS that is more flexible by introducing extra fuzzy rules, and by identifying other input variables which are sensitive to changes in system frequency. Also, the response of the FIS could be improved by making it predictive/adaptive to changes in system variables. The effect of droop will also have to be incorporated into the final design.

The FIS will have to be tested under more complex plant operating conditions, rather than just a single turbogenerator unit in frequency control mode. Also, the FIS will need to be implemented in hardware to test its effectiveness practically on the real plant. However, this paper shows that there is potential for improving the response of the turbogenerator governor by using FL techniques.

\section{Acknowledgements}

The authors would like to thank the First Hydro Company for their financial backing for this project, and for the useful help and advice given, the University of Abertay Dundee for the use of equipment and resources and the University of Bangor for their useful guidance, suggestions and assistance.

\section{References}

1. S.P. Mansoor, "Behaviour and Operation of Pumped Storage Hydro Plants", Thesis, University of Wales, Bangor, April 2000. 
2. M. Brown and C. Harris, "Neurofuzzy Adaptive Modelling and Control", Prentice Hall International (UK) Limited, Hemel Hempstead, Herts, 1994, p. 363.

3. R. Beale and T. Jackson, "Neural Computing - An Introduction", IOP Publishing Ltd., Bristol, UK, 1990.

4. O. Omidvar and D.L. Elliott, "Neural Systems for Control", Academic Press, San Diego, CA, USA, 1997.

5. E. Cox, "The Fuzzy Systems Handbook", Academic Press, Cambridge, MA, USA, 1994.

6. S. Goonatilake and S. Khebbal, "Intelligent Hybrid Systems", John Wiley \& Sons, Chichester, UK, 1995.

7. L. Jing, L. Ye and T. Zhou, "A New Intelligent Fuzzy Control System for Hydroelectric Generating Unit", Journal of Chinese Society of Electrical Engineering, Issue 1, 1998.

8. Y-S. Wang, G-C. Shang and T-X. He, "The PID-Type Fuzzy Neural Network Control and it's Application in the Hydraulic Turbine Generators", Proceedings of the 2000 IEEE Power Engineering Society Winter Meeting, Singapore, 23-27 January, 2000, Vol.1, pp. 338-343.

9. J. Lu, R.S. Ranganthan, G. Chen and H.A. Malki, "Predictive Fuzzy PID Control for Complex Processes", Proceedings of the 9th. IEEE International Conference on Fuzzy Systems - FUZZ-IEEE 2000, San Antonio, Texas, USA, 7-10 May, 2000, Vol. 2, pp. 544-548.

10. Y. Huang and S. Yasunobu, "A General Practical Design Method for Fuzzy PID Control from Conventional PID Control", Proceedings of the 9th. IEEE International Conference on Fuzzy Systems - FUZZ-IEEE 2000, San Antonio, Texas, USA, 7-10 May, 2000, Vol. 2, pp. 969-972.

11. "Fuzzy Logic Toolbox for use with MATLAB User's Guide Version 2", The MathWorks, Inc., Natick, MA, USA, 2000.

12. T. Bendell, G. Wilson and R.M.G. Millar, "Taguchi Methodology Within Total Quality", IFS Publications, Bedford, UK, 1990. 